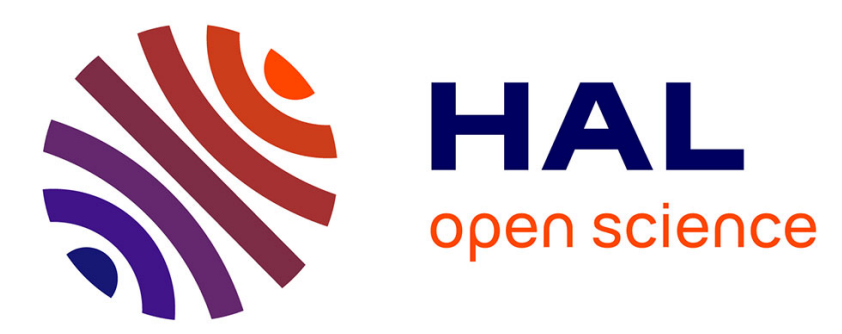

\title{
Travail collaboratif et information géographique pour l'enseignement secondaire
}

\author{
Thierry Joliveau, Sylvain Genevois
}

\section{To cite this version:}

Thierry Joliveau, Sylvain Genevois. Travail collaboratif et information géographique pour l'enseignement secondaire. Revue Internationale de Géomatique, 2008, 18, pp.531 - 548. 10.3166/geo.18.531-548. hal-01447618

\section{HAL Id: hal-01447618 https://hal.science/hal-01447618}

Submitted on 27 Jan 2017

HAL is a multi-disciplinary open access archive for the deposit and dissemination of scientific research documents, whether they are published or not. The documents may come from teaching and research institutions in France or abroad, or from public or private research centers.
L'archive ouverte pluridisciplinaire HAL, est destinée au dépôt et à la diffusion de documents scientifiques de niveau recherche, publiés ou non, émanant des établissements d'enseignement et de recherche français ou étrangers, des laboratoires publics ou privés. 


\title{
Travail collaboratif et information géographique pour l'enseignement secondaire
}

\author{
Thierry Joliveau $^{(1)}$ - Sylvain Genevois ${ }^{(1)}$ et (2) \\ (1) CRENAM-ISIG CNRS UMR 5600 \\ Université Jean Monnet de Saint-Etienne \\ 6 rue Basse-des-Rives \\ 42023 Saint-Etienne Cédex 2 \\ thierry.joliveau@univ-st-etienne.fr \\ (2) Equipe EducTice \\ INRP \\ 19 allée de Fontenay \\ BP 17424 - 69347 Lyon Cedex 07 \\ sylvain.genevois@inrp.fr
}

RÉSUMÉ. Les outils informatiques géographiques sont utilisés depuis plusieurs années à l'école. A partir de l'expérimentation d'une plateforme pédagogique collaborative en ligne organisée autour d'outils SIG et destinée aux élèves de Lycée, l'article tente de faire le point sur le nouveau contexte socio-technique qui accompagne le développement rapide des outils collaboratifs et personnels Web 2.0 du type globe virtuel. Une analyse des objectifs des techniques géomatiques à l'école, des modes de légitimité des outils et une réflexion sur la nature du travail collaboratif à partir de données géographiques conduisent à proposer les principes de plateformes éducatives de travail géocollaboratif.

ABSTRACT. Geospatial tools have been used for years at school. After a study assessment of an educational On-line GIS platform developed for high school students, this paper analyses the social and technical changes caused by the emergence of virtual globes and other Web 2.0 tools.It looks over what could be the aim of using geospatial techniques at school, how the tools are legitimated in an educational context and what is the nature of collaborative work based on geographical resources. In conclusion, some principles are proposed for designing platforms in order to achieve educational geocollaboration goals

мотS-CLÉS : géomatique, SIG, pédagogie, enseignement, géographie, Web 2.0, travail collaboratif.

KEYWORDS: GIS, education, teaching, geography, Web 2.0, geocollaboration

\section{Introduction}

Le développement des outils collaboratifs de type Web 2.0 («globes virtuels », blogs cartographiques, web collaboratif...) repose sur l'idée que de nouveaux outils peuvent contribuer à faire émerger une intelligence collective d'un problème. 
Comme le montre une enquête récente $^{1}$, ces nouveaux outils sont en cours d'adoption par les enseignants et les élèves : 52\% des enseignants d'histoiregéographie ont déjà utilisé Google Earth ou Google Maps avec leurs élèves, 34\% le Géoportail de 1'IGN et $80 \%$ déclarent avoir l'intention d'utiliser ces sites en classe dans les mois qui viennent. Au delà de l'effet de mode des «globes virtuels », ces chiffres témoignent du développement rapide des pratiques géomatiques en classe. En France comme dans de nombreux autres pays, l'introduction de la géomatique ${ }^{2}$ à l'école n'est pas un phénomène nouveau, elle a débuté dès le début des années 1990 avec l'expérimentation des premiers logiciels SIG éducatifs. Si les enseignants d'histoire-géographie sont encore peu nombreux à utiliser ce type de logiciel avec leurs élèves (moins de 10\% selon l'enquête), force est de constater qu'une large majorité des enseignants interrogés $(72 \%)$ souhaiteraient pouvoir échanger des séquences pédagogiques réalisées à partir d'outils géomatiques.

Ce texte tente de faire le point sur les évolutions en cours à partir d'un premier bilan d'une plateforme collaborative à destination des enseignants et des élèves du secondaire. Une analyse des bouleversements que l'Internet contributif produit dans l'enseignement conduit ensuite à une réflexion sur la place et le rôle des outils géomatiques à l'école et sur les enjeux de la géocollaboration, le travail en commun à partir de ressources géographiques dans le domaine éducatif. Nous terminons par une proposition des principales caractéristiques, qui doivent à notre sens guider la réalisation de nouvelles plateformes collaboratives.

\section{Premier bilan d'une plateforme collaborative à destination des enseignants et} des élèves du secondaire

\section{Présentation de GeoWebExplorer}

GeoWebExplorer ${ }^{3}$ est un prototype de plateforme collaborative développé pour aider les professeurs de l'enseignement secondaire français à mettre en œuvre des

${ }^{1}$ L'usage des outils numériques en Histoire-Géographie et en SVT, enquête nationale de l'INRP réalisée auprès de 862 enseignants de collège-lycée (du 10 janvier au 11 mars 2007). Pour lire le rapport complet d'enquête :

http://eductice.inrp.fr/EducTice/projets/geomatique/enquete2007

${ }^{2}$ Nous prenons le terme géomatique au sens large, comme un ensemble de technologies numériques pour acquérir, représenter et traiter l'information géographique. Elle comprend une panoplie d'outils, dont les logiciels de cartographie numérique, les Systèmes d'Information Géographique (SIG), les bases de données à références spatiales, les systèmes de télédétection et de modélisation numérique, les outils de localisation et de navigation en deux ou trois dimensions...

${ }^{3}$ La première version de l'application a été conçue au CRENAM/UMR EVS, en partenariat avec la société ESRI, par une équipe de géographes, géomaticiens et informaticiens constituée de Thierry Joliveau, Yvan Calcagni, Bernard Dupuis et Renaud Mayoud, dans le cadre du projet Gewed financé par le Ministère de la Recherche. Le code a été écrit par Yvan Calcagni. 
outils SIG dans le cadre de leur enseignement. La décision de son élaboration a été prise suite à la mise en œuvre d'un projet de recherche de l'INRP (Institut National de la Recherche Pédagogique), mené entre 2000 et 2004 [GEN 2003]. Dans ce projet, nous avons procédé à une expérimentation qui visait à utiliser des outils logiciels de type SIG dans le cadre de l'enseignement de la géographie en Lycée. Il était apparu dans ce projet qu'il fallait distinguer deux types de profil parmi les enseignants. Il existait d'une part en France un noyau de quelques dizaines d'enseignants passionnés et investis sur les questions de géomatique dans l'éducation. Ces experts utilisaient déjà plus ou moins régulièrement dans leur classe des outils professionnels tels que Mapinfo et ArcGIS, qu'ils avaient fait acheter par leurs établissements et appris souvent à utiliser en autodidactes. D'autre part, le reste des enseignants - une très grande majorité - restait beaucoup plus en retrait par rapport à l'utilisation de ces outils, et par rapport même aux Technologies de l'Information et de la Communication en général. Ce constat pouvait être modulé selon les disciplines enseignées. Les enseignants de Sciences de la Vie et de la Terre étaient plus habitués aux outils informatiques que les enseignants d'HistoireGéographie. Parmi ces derniers les enseignants ayant un cursus de géographie étaient plus ouverts à ces outils que ceux ayant une formation d'historiens. Ce projet avait permis aussi de repérer que, avant même toute question d'ordre pédagogique, l'usage de ces outils était fortement limité par les difficultés techniques et les contraintes matérielles que les enseignants rencontraient dans l'installation, la mise en œuvre et la maintenance des logiciels et des données nécessaires à leur enseignement.

L'idée était donc de fournir une solution clé en main accessible à distance, fournissant l'essentiel des ressources et permettant facilement à des enseignants non spécialistes d'effectuer des exercices en classe au moyen d'outils géomatiques simples. Il s'agissait de donner accès, à travers un simple navigateur, aux données et outils géomatiques nécessaires pour un usage en classe. Une solution fondée sur l'utilisation d'Internet libérait en effet l'équipe pédagogique des contraintes logistiques (disponibilité des salles, sécurité, maintenance des disques durs, organisation du libre accès des élèves, ...). Elle permettait aussi aux professeurs et éventuellement aux élèves d'avoir accès aux outils depuis chez eux. Bien sûr, il n'était pas envisageable que la préparation du jeu de données se fasse en ligne. Cela nécessiterait la mobilisation d'outils géomatiques offrant des fonctions de structuration de données (géoréférencement, projection, recalage, découpage, requêtage ...) qui, d'abord, sont relativement lourds et ensuite nécessitent des compétences spécifiques. L'idée était alors de s'appuyer sur le noyau des professeurs experts qui disposent des compétences pour construire ces jeux de données en local et de leur fournir la possibilité de les mettre à la disposition de tous les enseignants intéressés.

L'application fonctionne avec le logiciel serveur de données géographiques ArcIMS de la société ESRI. Elle a obtenu le prix du public aux Géos d'Or au Salon GéoEvénement en 2005 [JOL 2005], [JOL 2007]. 
Le concept central de GeoWebExplorer est ce que nous avons appelé un tutorat. Il s'agit d'un ensemble cohérent de cours, d'exercices assistés, de données et d'outils pour les traiter. Un tutorat suppose donc le choix d'un jeu de données et la sélection d'une collection d'outils adaptés au cours et aux exercices que l'enseignant propose. GeoWebExplorer permet au formateur de créer, de gérer et d'administrer en ligne des tutorats et de les mettre à disposition d'autres enseignants pour qu'ils s'en inspirent, les modifient et les enrichissent. Ces tutorats peuvent porter sur l'acquisition de notions ou de techniques de manipulation simples d'information géographique numérique. Mais l'application est plus directement orientée vers la construction de tutorats portant sur des questions de géographie, d'aménagement ou d'environnement, abordées au moyen de manipulations géomatiques simples. Les compétences informatiques demandées par GeoWebExplorer aux formateurs pour créer leur tutorat et aux élèves pour les exécuter, ne dépassent pas le niveau d'une maîtrise de la navigation sur le Web. La plateforme peut être utilisée dans une situation d'enseignement de type présentiel, directement dans la classe ou à partir d'une salle informatique. Elle peut être utilisée également dans une situation d'enseignement à distance, notamment pour pallier les problèmes d'accès à l'outil informatique dans les établissements.

\section{Aspects techniques}

GeoWebExplorer a été développé à partir du serveur de données géographiques ArcIMS de la société ESRI, sur le serveur Web Internet Information Server de Microsoft. Côté client, la solution ArcIMS permettait de construire facilement des tutorats utilisables avec le visualiseur intégré très simple ou avec des clients plus élaborés susceptibles de lire des services ArcIMS. Côté serveur, l'administration reste assez simple et l'ajout de nouveaux jeux de données (configuration de MapServices) est réalisable par un non spécialiste, ce qui était indispensable pour le projet, puisque les jeux de données transmis par les enseignants devaient être manuellement installés sur la plateforme.

Côté serveur, des modules de fonctionnalités de traitement d'information géographique ont été développés en langage ASP en utilisant le connecteur ActiveX d'ArcIMS. Le composant fonctionne avec des MapServices de type image. Côté client, les actions qu'effectue l'utilisateur au cours d'un tutorat (navigation dans le menu, rafraîchissement de tableaux, etc.) sont codées en JavaScript.

\section{Mise en ouvre}

La plateforme est installée à l'Université de Saint-Etienne ${ }^{4}$. Elle est fonctionnelle et a été mise en œuvre à titre expérimental pour les besoins internes de l'Université. Elle est utilisée dans les cours des étudiants de 2ème année de Géographie en

\footnotetext{
${ }^{4}$ Accès à la plateforme GeoWebExplorer : http://crenam56.univ-st-etienne.fr/geowebexplorer/
} 
initiation à la géomatique. A cet effet, divers tutorats ont été créés (initiation aux concepts géomatiques, limites d'un parc urbain, ...). Une présentation illustrée de ses fonctionnalités se trouve dans [JOL 2005]. Mais le prototype avait été conçu en premier lieu pour servir de support d'expérimentation pédagogique dans le cadre d'une activité de recherche. A l'heure actuelle, la plateforme est expérimentée par cinq professeurs associés à l'INRP sous la coordination de Sylvain Genevois dans le cadre d'une thèse de doctorat portant sur l'intégration de la géomatique dans l'enseignement de la géographie au second degré. Les études de cas proposées concernent des thématiques variées en lien avec les programmes d'enseignement en collège-lycée : la gestion des risques (cyclone Katrina), les problèmes de gestion et d'aménagement liés à l'eau (Schéma d'Aménagement et de Gestion des Eaux de la basse rivière d'Ain), le fait urbain et le phénomène de métropolisation (étude des métropoles à l'échelle de l'Union Européenne)...

\section{Un premier bilan de l'usage}

L'outil choisi a pris la forme d'une plateforme d'enseignement à distance fondée sur des techniques géomatiques pour différentes raisons. La solution s'appuie sur un serveur de données géographiques, car la donnée est au cœur de la ressource pédagogique et la gestion des droits d'accès en est facilitée. Les avantages d'une solution en ligne sont évidents. L'installation logicielle sur le poste de travail est minimale. L'outil est accessible partout et utilisable dans différents contextes (centre de documentation, domicile, cybercafés, ...). Le partage de ressources est facilité. La maintenance est banalisée. Les inconvénients sont tout aussi clairs : il faut disposer d'un accès Internet rapide et la gestion du système est nécessairement centralisée.

Les professeurs d'histoire-géographie qui ont utilisé GeoWebExplorer se déclarent globalement satisfaits de cette plateforme SIG, qui les affranchit de deux contraintes majeures que sont l'acquisition et l'installation de logiciel SIG d'une part, l'accès aux données géographiques d'autre part. Cependant des entretiens plus approfondis avec chacun des enseignants utilisateurs permettent de nuancer ce premier bilan d'usage. Certains d'entre eux ont ainsi rencontré des problèmes de réseau qui ont pu perturber le déroulement de l'activité pédagogique. Ces difficultés sont liées aux problèmes de maintenance des réseaux dans les établissements scolaires qui ne permettent pas de garantir une connexion Internet stable sur tous les ordinateurs et fiable dans le temps. Elles sont dues également à la maîtrise insuffisante des réseaux informatiques par les enseignants qui ont majoritairement une pratique de l'informatique sur poste individuel. Pour autant, il semble que les choses évoluent dans le bon sens : la lourdeur des authentifications qui faisait peur aux enseignants ne semble plus un problème aussi important. Les élèves eux-mêmes commencent à avoir l'habitude d'accéder à des sites web, avec login et mot de passe. Ce qui semble avoir davantage rebuté les élèves, c'est finalement l'interface un peu désuète de la plateforme qui n'avait pas l'aspect attractif des sites dynamiques que les élèves ont l'habitude de fréquenter sur Internet ! 
Outre les problèmes d'interface et d'ergonomie inhérents à un site Web, l'usage sur trois années de cette plateforme SIG a permis de dégager des pistes de réflexion concernant une utilisation dans un cadre pédagogique. Notons que la plateforme, conçue comme un prototype, n'a pas été testée au delà d'un petit cercle d'expérimentateurs. Bien que limité, son usage a malgré tout permis de montrer la gageure à vouloir surmonter tous les problèmes rencontrés par les enseignants dans l'utilisation de SIG. Certains d'entre eux découvraient la géomatique à travers GeoWebExplorer et devaient donc construire des tutorats en même temps qu'ils s'initiaient aux rudiments de la géomatique. D'autres enseignants, au contraire, étaient déjà rompus aux SIG, mais rencontraient des difficultés pour concevoir des jeux de données adaptés à leurs besoins pédagogiques. On perçoit ainsi toute la complexité de produire et de rendre accessibles des ressources SIG adaptées au contexte éducatif et à l'environnement pédagogique des enseignants et des élèves. Le système de tutorat qui était censé faciliter l'appropriation progressive des fonctionnalités et du jeu de données a pu, dans certains cas, s'avérer un frein à l'utilisation, en produisant des exercices trop longs et trop fermés, finalement rébarbatifs pour les élèves. Notons cependant que ce n'est pas le principe du tutorat qui est à blâmer. Celui-ci peut permettre de construire n'importe quel type d'exercice, du plus ouvert au plus fermé. C'est à l'enseignant de calibrer le caractère plus ou moins guidé des consignes qu'il souhaite donner à l'élève.

En conclusion de cette première partie, si GeoWebExplorer ne peut être considérée comme un modèle de plateforme pédagogique collaborative, le bilan de son utilisation expérimentale nous semble avoir démontré l'intérêt et la faisabilité d'un outil en ligne spécifique, permettant le partage et l'échange de ressources géomatiques entre enseignants. La diffusion de l'outil à une échelle plus large n'a pas encore été réalisée. Elle pose des problèmes moins conceptuels que techniques et organisationnels de dimensionnement de serveur et de maintenance d'une application sollicitée plus souvent. Mais, il est évident que le contexte général de l'usage de technologies géomatiques dans l'éducation a beaucoup changé depuis le moment de sa conception et qu'il est nécessaire d'analyser si la réponse apportée est encore adaptée.

\section{Un nouveau contexte socio-technique}

\section{Les globes virtuels}

Depuis la conception de la plateforme, plusieurs innovations sont apparues. La principale est bien entendu l'arrivée des globes virtuels. Google Maps, apparu en 2005, a très nettement bouleversé l'image des outils informatiques de visualisation de données géographiques chez les enseignants comme dans le grand public. Le succès des globes virtuels tels que Google Earth, Virtual Earth ou le Géoportail a été très important. Ils sont devenus des outils grand public, qu'un nombre croissant d'enseignants et d'élèves ont commencé à manipuler dans la vie quotidienne; ils 
sont rapidement entrés dans les classes où ils ont remplacé la carte murale, l'atlas ou les illustrations des manuels.

Une recherche rapide des séances pédagogiques disponibles sur Internet permet d'ores et déjà de repérer une cinquantaine de séances s'appuyant sur l'utilisation d'un globe virtuel (dont plus de $80 \%$ avec Google Earth et seulement 5\% avec le Géoportail). Les modes de mise en œuvre pédagogique sont très variés, de la simple géovisualisation à des formes d'exploration géographique, voire d'immersion dans la réalité virtuelle en 3D. La diversité des usages témoigne en tous les cas du potentiel pédagogique de ces outils de géolocalisation sur Internet et de la rapide familiarisation des professeurs avec ces outils grand public. Si tous les enseignants n'ont pas été convaincus, une part significative de ceux qui n'étaient pas prêts à utiliser des outils SIG, a adopté ce type d'outils, mais aussi les pratiques qui leur sont associées. L'image des outils SIG a été aussi transformée : la part assignée aux processus de géovisualisation et d'interprétation de ce qui est vu a tendance à devenir plus importante que celle accordée traditionnellement à l'analyse et au traitement de l'information géographique.

\section{Le Web 2.0}

Google Maps est l'application emblématique de ce que l'on appelle le Web 2.0. L'appellation est controversée car le Web 2.0 ne peut être compris vraiment ni comme une innovation technologique, ni comme une innovation économique. Pourtant il serait à notre sens erroné de n'y voir qu'un concept marketing ou une idée de journaliste. Web 2.0 est un mot qui vient nommer quelque chose qui est dans l'air et que l'on peut résumer par l'idée que nous sommes en train de passer d'un Web de consultation à un Web de contribution. Un des moteurs du Web devient la production de contenu par les utilisateurs eux-mêmes au moyen de nouveaux outils d'écriture et d'édition utilisables individuellement et collectivement. Les outils estampillés Web 2.0 (pour aller vite : les plateformes de blogs, de wikis, de réseaux sociaux) correspondent en effet à des niveaux de contribution et de collectifs différents. Au niveau le plus simple, chacun contribue individuellement mais sans but commun (blogs ou sites contributifs comme Flickr ou Youtube). A un deuxième niveau, les échanges entre contributions individuelles se structurent sous forme de liens dans des réseaux plus ou moins ouverts, à travers les forums, les logiciels de réseau social (Myspace, Facebook, LinkedIn, ...). Enfin des Wikis correspondent à l'émergence d'un but commun et d'une certaine coordination. En fait les pratiques sont plus subtiles, car même une activité individuelle sur un blog peut conduire à travers des systèmes de rétroliens à organiser et partager les contenus. Dans le domaine géographique on peut citer deux applications emblématiques du Web 2.0 : le projet Openstreetmap http://www.openstreetmap.org/ qui s'est donné comme objectif de contribuer à la construction collaborative d'une carte du Monde libre de droits. Les participants créent des objets spatiaux à partir de levés de terrain sous forme de traces GPS et de photographies. Wikimapia (http://www.wikimapia.org/) 
est un projet du même type, qui consiste annoter les vues satellitaires de Google Maps au moyen d'un système wiki.

Le Web 2.0 s'accompagne d'une culture contributive. On peut penser que les élèves ont déjà en partie intégré cette culture à travers les blogs et leur pratique assidue des forums. Les enseignants les plus jeunes ont commencé à la pratiquer.

\section{L'individualisation de la géomatique}

Google Maps en soi n'est pas au départ une application fondée sur la contribution des utilisateurs. C'est le service Google « My Maps » (Mes cartes) lancé en avril 2007 qui permet à chaque utilisateur de créer des cartes personnalisées avec ses annotations, de les partager avec d'autres utilisateurs et d'ajouter des cartes interactives et des outils créés par d'autres utilisateurs. Mais l'API Google Maps a été citée par O'Reilly dans le texte [ORE 2005] qui a contribué en 2005 à lancer le Web 2.0 comme le composant type permettant de créer des services collectifs. Une autre innovation est en effet l'apparition des services géolocalisés, basés souvent sur les API (Application Programming Interface) des différents globes virtuels, et qui permettent de localiser des ressources sur une base géographique. Toutes ces nouvelles activités sont impulsées par de nouvelles sociétés dynamiques et innovantes (Weogeo, Geocommons, Metacarta, NavX, Dismoiou, ...). Mais les principaux opérateurs de ces nouvelles applications sont les colosses de l'Internet : Google, Microsoft, Yahoo... On assiste à l'émergence d'une géomatique personnelle, dont l'utilisateur cible est l'individu qui cherche à organiser spatialement son monde personnel. On l'aide à se repérer, se diriger, planifier ses déplacements, évaluer son environnement, estimer les ressources disponibles localement, visualiser les lieux à distance, gérer sa famille, jouer, laisser des traces pour ses amis et retrouver celles qu'ils ont laissées, etc.

Les techniques employées sont aussi nouvelles. Le développement se fait au moyen d'applications composites (mashup) et de microcomposants logiciels intégrables à des sites Web (widgets) plus que sous la forme de logiciels classiques. De nouveaux standards de format de données sont apparus liés à Google, comme le Keyhole Markup Language (KML), ou au GPS comme le GPX (GPS eXchange Format). Les dispositifs matériels de saisie sont ceux dont dispose le grand public : GPS personnels, appareils photo numériques, téléphones. La géolocalisation par les moyens les plus divers (adresse IP, bornes Wifi, GSM, flux GéoRSS...). Les développeurs sont issus du monde du logiciel libre, mais ont rarement une culture géomatique à l'origine.

Le développement de cette géomatique personnelle ne concernera pas indifféremment tous les enseignants et tous les élèves. Mais il n'est pas raisonnable de penser que les pratiques géomatiques à l'école ne seront pas influencées par la généralisation du GPS et de la géolocalisation par téléphone. 


\section{Les innovations dans le secteur de l'éducation}

Les plateformes de travail collaboratif en ligne se sont multipliées (Google Documents, Zoho). Il devient aisé de placer ses données sur Internet, mais aussi d'y accéder avec des outils situés eux aussi en ligne. Cela concernait des applications simples comme le traitement de texte et les tableurs, mais cela s'étend à des outils plus complexes. Un site comme Zoho.com propose ainsi la conception et le partage des bases de données ou la création d'applications. Si cela inquiète encore certains utilisateurs, pour des raisons évidentes de confidentialité et de sécurité, c'est une tendance en phase avec le besoin de garder accès à ses ressources, quel que soit l'endroit où l'on se trouve.

Dans le secteur de l'éducation, les plateformes éducatives en ligne adaptées au travail de l'enseignant connaissent aussi de fortes évolutions. Même si elles ne sont pas encore utilisées de manière systématique, les plateformes éducatives arrivent dans les collèges et lycées sous différentes formes, soit à l'initiative de quelques enseignants qui partagent leurs travaux sur des sites contributifs, soit à l'initiative de collectivités territoriales qui équipent systématiquement les établissements scolaires d'Envionnements Numériques de Travail (ENT). Dans ce dernier cas, il peut s'agir d'équipement informatique de grande ampleur. Tous les membres de la communauté éducative (parents, élèves, enseignants) accèdent via Internet à un portail de services et peuvent ainsi partager des informations et des applications au sein d'un même établissement. Pour l'instant, ces ENT concernent en majorité l'accès à des services administratifs (messagerie, gestion des salles, saisie des notes, ...), mais ils s'enrichissent progressivement de ressources pédagogiques (cahier de texte numérique, cours et exercices en ligne, liens vers des sites web éducatifs...). L'objectif à terme est de pouvoir disposer d'un identifiant unique qui permettra à l'utilisateur, au sein d'un même espace intégrateur, d'avoir accès à des ressources et à des applications pédagogiques, notamment celles qui concernent l'information géographique.

Le projet Edugéo ${ }^{5}$ est aussi une initiative significative dans le domaine qui nous intéresse. Il s'agit d'un service conçu par l'IGN en partenariat avec le Ministère de l'Education Nationale. Il s'adresse aux élèves et aux professeurs des établissements d'enseignement public. Plusieurs services sont associés : consultation d'informations cartographiques : cartes au 1/25 000 scannées, images satellites, photos aériennes et cartes anciennes, espace de téléchargement de données spécifiques, fourniture d'un outil de croquis et de fonctions SIG simples, Wiki. Le fait que le service ne soit accessible que sur abonnement et utilisable seulement dans l'établissement et que les ressources ne concernent que l'espace français risque de limiter son usage. Ce projet est bien le signe de l'intérêt croissant que connaissent les plateformes éducatives dans ce domaine. On peut se demander pourtant si une solution unique, générale et impulsée par l'Etat central est la bonne réponse à la multiplication et diversification

\footnotetext{
${ }^{5}$ Edugéo, le service éducatif du Géoportail : http://www.edugeo.fr/
} 
des besoins, des pratiques et des initiatives. Des initiatives décentralisées et issues de regroupements de professeurs commencent à proposer, sur un mode collaboratif, des ressources pédagogiques très intéressantes (voir par exemple, pour les Sciences de la Vie et de la Terre, le LaboSVT.com : http://www.labosvt.com.)

\section{Vers des plateformes éducatives de travail géocollaboratif}

Il est donc nécessaire de revoir la conception des plateformes collaboratives à la lumière des bouleversements en cours, que ceux-ci soient purement techniques, liés aux modes d'accès aux données ou à des changements dans les pratiques. A la lumière de l'expérience GeoWebExplorer, il nous semble qu'il est possible de réfléchir aux conditions que des plateformes de ce type devront remplir.

Il faut d'abord éclaircir les objectifs pédagogiques de la plateforme. GeoWebExplorer avait été conçue sur la base d'une césure épistémologique entre la géomatique et les sciences d'application. Il semblait nécessaire de maîtriser des concepts géomatiques avant de les appliquer en géographie et en SVT. Ce postulat de principe doit être questionné.

Dans la continuité du point précédent, il faut réinterroger aussi le modèle de légitimation des outils utilisés. GeoWebExplorer visait à mettre à la disposition des enseignants un outil géomatique de type professionnel, adapté pour être utilisable par des élèves. Cette position est-elle encore valide, maintenant que sont apparus des outils comme les globes virtuels qui se situent à la frontière entre les outils professionnels SIG et les outils personnels?

GeoWebExplorer était conçue comme un outil autonome et fermé qui prenait en charge toutes les fonctions du système : base de données, outils d'interrogation et d'analyse, centrale d'échange. Cette approche est-elle encore pertinente, alors que l'univers du Web lui-même devient à la fois éclaté et collaboratif ?

\section{La nature de l'objectif pédagogique}

On doit distinguer trois options [SUI 1995] : on peut enseigner spécifiquement les méthodes et outils de la géomatique, entendue comme la technoscience de l'information à référence spatiale ; on peut enseigner comment appliquer certaines méthodes et outils de la géomatique dans un domaine spécifique (ex. : l'hydrologie ou la géographie) ; on peut enseigner une discipline ou un domaine thématique en utilisant des méthodes et outils géomatiques. Dans le contexte de l'enseignement secondaire de la géographie ou des SVT, nous ne sommes clairement pas dans une situation conduisant à choisir la première option. Il ne s'agit pas de former à la géomatique en tant que telle. Mais la distinction entre la deuxième et la troisième situation peut faire débat. Certes, dans l'enseignement secondaire, la géomatique est le moyen et non l'objet de l'apprentissage. Mais est-ce une bonne chose de rendre 
les concepts de la géomatique complètement transparents lorsque l'on traite une question de géologie ou de géographie ?

La philosophie de GeoWebExplorer relevait de la deuxième option proposée par Sui. A la base était l'idée qu'il existe une méthodologie intrinsèque géomatique, et qu'il n'était pas pertinent de tenter de s'en abstraire, même dans l'enseignement secondaire. Il semblait au contraire nécessaire de donner conscience à l'élève ou à l'enseignant des différences conceptuelles fondamentales entre les trois éléments d'un Système d'Information Géographique : la base de données géoréférencées à laquelle il a accès, les outils matériels et logiciels dont il a besoin pour gérer ces données, et l'ensemble des méthodes et concepts qu'il doit maîtriser pour mettre en œuvre ces outils avec les données. Il semblait donc nécessaire que l'utilisation de la plateforme supporte une initiation des enseignants et des élèves aux notions de projection, de systèmes de coordonnées, de couches associées aux tables, de requêtes attributaires et spatiales, de symbologie et de discrétisation, pour ne citer que certaines notions. Des tutorats étaient d'ailleurs réservés à l'exploration de ces notions. Cette volonté d'expliciter les apprentissages géomatiques auprès des élèves de l'enseignement secondaire s'expliquait par le fait qu'un des objectifs était d'acclimater une discipline extérieure dans les pratiques éducatives du Lycée.

A l'inverse, certains enseignants, et pas seulement les plus réfractaires aux outils informatiques, pensent que les concepts géomatiques sont secondaires et qu'il est nécessaire de les rendre invisibles, en encapsulant les outils appropriés dans des exercices de géographie ou de géologie. Cette alternative n'en est pas forcément une. On peut envisager une continuité de situations pédagogiques qui vont de la géomatique pure à l'application pure et dans lesquelles les concepts sont plus ou moins visibles. Toutefois, la conception d'une plateforme collaborative répondant à un tel cahier des charges en est complexifiée.

L'arrivée des globes virtuels a un peu changé la donne de ce point de vue. Pour les géomaticiens professionnels, leur utilisation s'accompagne en effet d'une certaine dégradation conceptuelle. Certes, les globes virtuels offrent une interface simplifiée et intuitive pour rapidement naviguer dans l'information géographique. Mais ils doivent leur efficacité au fait qu'ils sont essentiellement des infrastructures de bases de données équipées d'interfaces simples pour visualiser et interroger ces bases et leur ajouter des jeux simples de données. Les données sont subsumées dans l'outil de consultation. On voit apparaître chez les utilisateurs néophytes une confusion entre la base de données (images aériennes, cartes raster) et les outils pour les visualiser (interface Google Maps, Google Earth ou Virtual Earth). Les données sont essentiellement au format image, sans attributs associés. L'utilisateur ne perçoit pas la structure de la base de données dont il dispose et n'a pas accès à des métadonnées correctes. Les fonctions restent très simples : pas de requête, pas de symbolisation, pas de calcul, pas d'analyse spatiale formalisée. Seule l'analyse visuelle est possible. Les globes virtuels amènent pourtant de nombreux élèves et enseignants à utiliser l'information géographique d'abord individuellement, mais aussi de plus en plus souvent dans des tâches collectives en classe. Ils contribuent aussi à diffuser une 
connaissance des outils simples de navigation (notions de couche, de zoom, de panoramique, d'actions de cacher/montrer les couches, de gérer la transparence des couches, etc., toutes tâches qui sont les fonctions de base de GeoWebExplorer). Bien que ne relevant ni de la géomatique professionnelle ni de disciplines scolaires comme les SVT ou la géographie, les globes virtuels ont leur place dans la panoplie des outils de l'information géographique utilisables dans un cadre pédagogique.

Ceci étant dit, le contexte du projet de la géomatique a lui-même évolué ces dernières années. La problématique des SIG, entendus comme Systèmes d'Information à part entière, qu'ils répondent aux besoins de territoires, d'institutions ou d'entreprises reste d'actualité. Mais de nouvelles questions ont émergé : la thématique de la localisation et des services liés à la mobilité des personnes et des biens, l'émergence du Géoweb (ou Geospatialweb), qui vise à associer une caractéristique géographique à tout bloc d'information placé sur Internet. Il faut aussi prendre en compte le processus rapide, généralisé et systématique de numérisation et de géoéréférencement des documents stockés dans les bibliothèques, les collections, les musées, les archives... [HIL 2006]. Autant de défis que la géomatique doit relever en proposant des solutions pertinentes prouvant sa capacité à cumuler des savoirs et des méthodes efficaces. Les débats actuels sur le Net à propos de la Neogeography peuvent ainsi s'interpréter pour partie comme une mise en cause de la capacité de la géomatique traditionnelle à répondre aux questions émergentes des services individuels et collectifs liés à la localisation des personnes.

\section{La légitimation des outils}

La question de la nature de la géomatique n'est pas indifférente dans le choix des outils et applications pédagogiques. En effet pour qu'un outil soit utilisé à l'école, qu'il s'agisse d'un SIG ou d'un globe virtuel, il faut qu'il soit considéré comme légitime. Nous avons dénombré quatre principaux modes de légitimation.

La première légitimation se fait par l'usage social. Dès lors qu'il est utilisé dans la société, il faut que les élèves soient initiés à un usage réfléchi et raisonnable d'un outil. C'est ce qui s'est passé avec l'Internet et ce qui va se passer avec les globes virtuels ou les GPS. Dans ce cas cependant, il faut établir une rupture entre l'usage quotidien et son usage à l'école, en développant des usages spécifiquement scolaires des outils. Connexe à cette forme de légitimation est le registre de la citoyenneté. Si la participation à la vie publique nécessite de disposer de certains outils, c'est à l'école de prendre en charge la formation à ces outils. C'est donc la question de la citoyenneté géographique qui est posée. Dans ce contexte, l'introduction de la géomatique professionnelle était perçue comme un enjeu démocratique pour de futurs citoyens qui auraient à comprendre des cartes réalisées par informatique. Cette légitimité s'affaiblit si l'on pense que la consultation des cartes se fera par l'intermédiaire des interfaces simplifiées que sont les globes virtuels. En revanche, la diffusion des outils de la géomatique personnelle à l'école doit se faire accompagner de modes d'usage spécifiques et garder une dimension critique. 
Le deuxième mode de légitimation est professionnel ; l'outil doit être un outil réellement utilisé par des hommes de métier. C'est ce que de nombreux acteurs avaient en tête au début des années 2000 quand ils défendaient l'usage dans les classes des logiciels SIG commerciaux (Arcview, Mapinfo, Géoconcept...), nonobstant leur caractère un peu difficile d'accès. Cette légitimation semble fragile car il n'est pas question de former des géomaticiens dans l'enseignement secondaire. Ce mode transparaît aussi dans la volonté de transformer l'image de la discipline scolaire qui accompagnait l'entrée des SIG à l'école. Les SIG étaient, pour les enseignants qui les promouvaient, le symbole d'une géographie active, pratique, débouchant sur de vrais métiers ...

Le troisième mode de légitimation est pédagogique. L'outil ne doit pas être une fin, mais un moyen. Il ne doit pas être un détour pour l'apprentissage des notions du cours. Dans ce sens, il doit y avoir une intégration pédagogique et didactique de l'outil dans l'enseignement de la discipline scolaire. Par exemple l'intégration d'outils géomatiques dans les classes en 2002-2003 conduisait à mobiliser des notions un peu oubliées des programmes comme les systèmes de projections et de coordonnées et des objets inusités comme les cartes topographiques. Pour certains les SIG symbolisaient une géographie archaïque, plus à même de décrire les phénomènes qu'à les conceptualiser pour les expliquer. Les outils valent principalement par la qualité des concepts qu'ils aident à transmettre. C'est donc d'abord une question de programme : que faut-il enseigner? Si l'on admet que ce qui est au cœur des apprentissages contemporains c'est la question de l'utilisation de l'information, y compris dans sa dimension spatiale, pour raisonner valablement sur un phénomène, il faut que les outils mis en œuvre permettent de comprendre et de critiquer les modes de production de cette information, ses limites de validité, ses méthodes d'analyse et de communication.

Le quatrième facteur de légitimation est technique. Il faut qu'élèves et enseignants parviennent à une maîtrise pratique de l'outil. En France, les Technologies de l'Information et de la Communication (TIC) font partie désormais du "socle" commun de connaissances à l'école. Avec le Brevet Informatique et Internet (B2i) et le Certificat Informatique et Internet (C2i), élèves et enseignants sont censés maîtriser ces nouvelles technologies, en avoir un usage critique et raisonné. Même si elle n'est pas encore gommée, l'inversion des compétences que l'on a souvent constatée entre enseignants et élèves [JOL 2005] et qui contribue à freiner le développement des outils numériques va se combler progressivement.

Ces modes de légitimation sont contradictoires. Un outil professionnel n'est pas nécessairement pédagogique. Par ailleurs, si l'école n'a pas vocation à employer tous les outils utilisés quotidiennement pour raisonner valablement sur un phénomène, elle ne peut pas non plus les ignorer par principe. Les pratiques sociales de référence jouent un rôle majeur dans la légitimation des contenus et des techniques à enseigner, sans pour autant remettre en cause l'importance des savoirs scientifiques et didactiques. 


\section{La dimension collaborative}

La dimension collaborative de GeoWebExplorer reste encore limitée. Elle concerne d'abord les enseignants en leur permettant d'organiser le partage de tutorats et en facilitant le travail collectif d'amélioration d'un tutorat commun. Elle gère aussi la relation entre enseignants et élèves en fournissant des leçons, des exercices et des contrôles. En revanche la plateforme ne prend pas en charge le travail collaboratif entre élèves, ni d'ailleurs un véritable travail collaboratif conjoint enseignants-élèves.

Selon Dillenbourg [DIL 1999], il convient d'ailleurs de distinguer le travail collaboratif, qui suppose la création d'une œuvre commune, du simple travail coopératif qui repose seulement sur le travail en groupe sans réalisation d'une œuvre commune. Pour l'instant, la plateforme GeoWebExplorer en reste au niveau le plus simple, celui du travail coopératif. Nous sommes encore loin du niveau collaboratif qui supposerait la construction commune d'un tutorat par les enseignants ou la résolution de problème et la recherche en commun de réponses par les élèves. Le travail coopératif intervient principalement à deux niveaux :

- au niveau des formateurs, à travers le partage de scénarios pédagogiques : chaque formateur est libre de mettre le scénario qu'il a créé à disposition des autres formateurs. L'éditeur de scénarios fonctionne ainsi comme une banque de ressources et d'activités mutualisables au sein d'une communauté d'utilisateurs.

- au niveau des apprenants, à travers l'apprentissage coopératif, au sein du groupe classe ou par groupes d'apprenants : l'apprenant est autonome et responsable de ses apprentissages, il est également motivé pour participer à l'apprentissage des autres.

Appliqué à la géomatique, le travail collaboratif débouche sur la notion de géocollaboration [MAC 2005]. Comme l'ont montré Pornon et al. [POR 2007], la géocollaboration ne se limite pas à la collaboration assistée par ordinateur, elle implique des aspects sémantiques et cognitifs. L'usage collaboratif d'outils géomatiques mobilise de nouvelles compétences et met en jeu de nouveaux savoirs et savoir-faire, tel que l'élaboration et le partage de connaissances géographiques, la construction d'outils cognitifs pour se représenter collectivement l'espace...

La plateforme GeoWebExplorer ne permet pas d'interagir à distance pour résoudre collectivement des problèmes géographiques. Cependant elle constitue un premier stade dans le partage de données et dans la géocollaboration, dans le sens où elle favorise les interactions entre utilisateurs qui accèdent à des tutorats partagés. Elle concerne une petite communauté d'utilisateurs, sans qu'il soit pour autant possible de parler d'une véritable communauté de pratique [WEN 2000]. Pourtant la plateforme permet à ses usagers de partager des fonctions, des activités et des données au sein d'un environnement intégrateur. Son utilisation s'inscrit également 
en lien avec une communauté de pratique plus large, qui est celle des membres de l'Observatoire de pratiques géomatiques de l'INRP'.

\section{Eléments pour une plateforme éducative de travail géocollaboratif}

Passée au filtre des questionnements ci-dessus, on mesure combien GeoWebExplorer est une réponse encore modeste aux enjeux du travail collaboratif en ligne. En revanche certains de ses principes sont vraisemblablement à conserver. Nous voulons tracer maintenant les grandes lignes des concepts qui pourraient présider à la création d'une plateforme répondant aux exigences que nous venons de présenter.

Il faut d'abord diversifier les ressources géomatiques que la plateforme devrait distribuer. Ces ressources seraient au nombre de quatre : les activités, les données, les fonctions de traitement et les notions à enseigner. Il nous semble important de conserver le principe à la base de GeoWebExplorer, qui est de proposer une organisation cohérente entre les différentes ressources utilisées. L'élément intégrateur de la plateforme est donc l'activité que le pédagogue veut réaliser avec ses élèves et qui doit combiner toutes les autres ressources (cela correspond à une extension de la notion de tutorat produit par l'enseignant).

La plateforme donne donc accès à une liste d'activités, de fonctions, de données et de notions. Toutes ces ressources peuvent être proposées par les enseignants pour leur usage propre ou pour une mise à disposition collective. La description de toutes ses ressources devrait être aussi un travail collaboratif.

Les activités correspondent aux tutorats de GeoWebExplorer. Elles sont enrichies d'attributs descriptifs (public visé, durée, objectifs pédagogiques, mode de mise en œuvre) et renvoient aux autres ressources qu'elles mobilisent. De manière à les rendre plus collaboratives (construction d'une activité en commun) que simplement coopératives (partage et réutilisation d'activités), un système de wikis, blogs ou forums devra être proposé pour construire les activités à partir des ressources de la plateforme.

Les fonctions : dans GeoWebExplorer, c'était la seule ressource que le concepteur du tutorat pouvait réellement choisir. Ce qu'on appelle une fonction est un traitement élémentaire d'information. Si un descriptif exhaustif de tous les traitements possibles de l'information géographique n'est pas possible, celui des fonctions élémentaires utilisables avec les élèves du secondaire est envisageable. Un inventaire a déjà été réalisé avec les étudiants du Master SIG de Saint-Etienne et structuré sous forme de Wiki (voir: http://mastersig.free.fr/wiki/doku.php). Un certain nombre de fonctions de base seraient disponibles sur la plateforme, comme c'est déjà le cas dans GeoWebExplorer. D'autres seraient accessibles par des outils

${ }^{6}$ Pour accéder à la liste de diffusion et aux travaux de l'Observatoire de pratiques géomatiques de l'INRP : http://eductice.inrp.fr/EducTice/projets/geomatique/ 
comme les globes virtuels ou des outils SIG disponibles en local. Une contrainte est que la description des fonctions de ces outils soit compatible avec le cadre fonctionnel de la plateforme. Les standards d'interface de service web WPS (Web Processing Service) que l'Open Geospatial Consortium a approuvés il y a quelques mois devraient faciliter la publication et la documentation de géotraitements accessibles sur le Web et donc mobilisables aisément par une plateforme de ce type.

Les données : il s'agit aussi d'une ressource qui, comme dans GeoWebExplorer, doit être choisie par le concepteur du tutorat. Mais il faut que les jeux de données soient segmentables, par couches ou collections d'entités. Les jeux de données peuvent être chargés puis distribués sur la plateforme, si les droits le permettent. Il peut aussi s'agir de liens vers des services de données normalisés, type WMS ou WFS, ou de références aux géoportails ou autres globes virtuels. Pour être intégrées sur la plateforme, les données doivent bien entendu disposer de métadonnées.

Les notions sont géomatiques ou disciplinaires. Elles doivent être explicitées. Un outil de type Wiki devrait permettre de travailler collaborativement sur les définitions et limites de ces notions.

La normalisation et la diffusion des plateformes d'enseignement à distance conduisent à étudier la manière dont pourront s'interfacer une plateforme collaborative de ce type avec les plateformes éducatives classiques. Tout ce qui concerne la gestion des classes et les authentifications pourrait être confié à ces plateformes généralistes qui font cela très bien. De la même manière, il faut étudier comment les activités pourront être mises à un format standard (SCORM par exemple) pour qu'elles soient intégrables facilement dans des plateformes généralistes. La principale difficulté pour l'intégration dans une plateforme généraliste reste le caractère spécifique de ces activités, fonctions et données lié à leur dimension spatiale.

\section{Conclusion}

La problématique de la géomatique dans l'éducation a largement évolué ces dernières années. On est passé d'une recherche concernant un logiciel SIG didactique à la construction d'un outil pour mettre en œuvre des méthodes géomatiques variées dans un contexte pédagogique plus diversifié. Les pratiques se sont extrêmement diversifiées et individualisées, au point qu'il semble peu réaliste d'envisager une solution unique et générale.

Par ailleurs, les évolutions techniques sont devenues tellement rapides qu'il semble illusoire de construire une représentation stable de ce que devrait être l'utilisation de la géomatique en classe. Celle-ci ne peut être, récursivement en quelque sorte, que le résultat d'un travail collaboratif de communautés d'enseignants. Ce sont les plateformes correspondant aux besoins et attentes de ces 
communautés qui sont à inventer, en prenant soin d'associer ces dernières à leur conception et à leur usage.

Remarquons en conclusion que les outils de type SIG collaboratif en ligne sont loin d'être le seul apport que la géomatique peut apporter au domaine éducatif. La diffusion des outils individuels de localisation ouvre la voie à des formes d'activité pédagogique fondées sur la collecte d'information par les élèves directement sur le terrain. Des jeux éducatifs combinant des déplacements dans un espace réel et des techniques de réalité augmentée ont déjà été testés. Un renouvèlement complet de la problématique est perceptible à un horizon plus ou moins lointain. Mais il reste encore beaucoup de travail pour disposer d'outils collaboratifs simples, efficaces et accessibles dans les conditions classiques du travail en classe.

\section{Bibliographie}

[DIL 1999] Dillenbourg P. (1999) What do you mean by collaborative learning?. In P. Dillenbourg (Ed) Collaborative-learning: Cognitive and Computational Approaches. (pp.1-19). Oxford: Elsevier

[GEN 2003] Genevois, S., Carlot Y., Joliveau T., et al. (2003). Le SIG : un outil didactique innovant pour la géographie scolaire. Dossiers de l'ingénierie éducative, $n^{\circ} 44$, p10-13

[HIL 2006] Hill, L. (2006). Georeferencing. The Geographic Associations of Information. Boston, The MIT Press. 272 p.

[JOL 2005] Joliveau, T., Calcagni Y. et Mayoud R. (2005). Geowebexplorer, un outil géomatique collaboratif au service des enseignants et des élèves. Actes de Géoforum, Lille 2005 "Savoir penser et partager l'information géographique les SIG", Lille, Géographes associés AFDG, p169-178

[JOL 2007] Joliveau, T. et Genevois S. (2007). Une plate-forme pédagogique collaborative pour enseigner la géographie en lycée. Analyse, principes et mise en oeuvre. Conférence Québéco-Française pour le Développement de la Géomatique (CQFD-Géo), SAGEO 2007, Rencontres internationales Géomatique et territoire, Clermont-Ferrand. http://www.emse.fr/site/SAGEO2007/CDROM/CQFD09.pdf

[MAC 2004] MacEachren, A.M. Brewer, I. (2004). Developing a conceptual framework for visually-enabled geocollaboration, International Journal of Geographical Information Science 18(1), p 1-34

[POR 2007] Pornon, Henri, Noucher Mathieu (2007). Bilan et perspectives de 20 années de géomatique. Vers des SIG plus collaboratifs. Les communautés de pratiques, Géomatique Expert $n^{\circ}$ 59, octobre-novembre 2007.

[ORE 2005] O'Reilly, T. (2005). "What is Web 2.0. Design Patterns and Business Models for the Next Generation of Software", consulté le 09/05.2008 : http://www.oreillynet.com/pub/a/oreilly/tim/news/2005/09/30/what-is-web-20.html 
18 Géomatique. Volume $18-\mathrm{n}^{\circ} 4 / 2008$

[SUI 1995] Sui, D. Z. (1995). A Pedagogic Framework to Link GIS to the Intellectual Core of Geography. Journal of Geography(94(6)): 578-591

[WEN 2000] Wenger, Etienne (2000). Communities of Practice. Learning, Meaning and Identity. Cambridge University Press, 311 p.. 